\title{
Thermo-prevention of commons colds: the Messini method. A proposal for a new and simple form of individual prevention
}

\author{
Sergio Messini ${ }^{1}$ \\ ${ }^{1}$ Woman Health Center
}

April 28, 2020

\section{Introduction}

Upper respiratory tract diseases and colds in the first place (common cold) are considered trivial diseases, which, almost inevitably, are encountered seasonally in winter/cold periods.

In most cases, they run benevolently, with self-resolution in five to seven days.

However, serious complications may be present, especially in elderly or chronically ill subjects; or there may be viral variants of greater aggression (SARS-CoV; MERS-CoV; SARS-CoV-2)

However, even if only considering the most cases, i.e. those with a benevolent course, the social and economic impact is remarkable.

\section{Economic aspect}

The economic impact of the common cold is enormous. ${ }^{1,2}$

The University of Michigan (USA) Health System (UMHS) estimates in 2003, that more than 62 million cases of common cold each year require medical attention in the United States or limit the activity of the affected people.

Every year, the common cold causes 45 million days of lost work, 22 million lost school days and an overall economic impact of more than $\$ 20$ billion.

Each year over 100 million medical visits can be attributed to the common cold in the USA, with an estimated cost of at least $\$ 7 \cdot 7$ billion a year.

An additional cost of $\$ 2.9$ billion is incurred annually for the purchase of over-the-counter drugs intended for the treatment of cold symptoms and an additional $\$ 400$ million for the purchase of prescription drugs for symptomatic alleviation.

In addition, more than $\$ 1 \cdot 1$ billion is spent annually on 41 million antibiotic prescriptions targeting colds, although antibiotics have no effect on a viral disease.

\section{Aetiology and pathogenesis}

Common cold diseases are due to multiple viruses, affecting the upper respiratory tract ${ }^{3}$

Human Rhinovirus (HRVs ) 30-80\% (a genus ofPicornaviridae with 99 known serotypes); Coronavirus1015\%; Orthomyxovirus 05-15\%; Coxsackievirus; Echovirus; Paramixovirus (Human Parainfluenza Virus); Human respiratory syncytial virus (SRV); Adenovirus; Enterovirus; Metapneumovirus. In addition, over 200 viruses associated with common cold diseases are known and often more than one viral species is involved in the infection. 
These viruses penetrate the mucosal layer of the nasal or bronchial mucociliary epithelium; this involves damage to the hair cells and a consequent release of bradykinin, prostaglandin, histamine and cytokines. These mediators promote the inflammatory response thus causing inflammation of the nasal tissues, detachment of hair cells and alterations of epithelial integrity. As a consequence, the subject sneezes and has a runny and stuffy nose. The mediators themselves are also responsible for laryngo-pharyngitis (sore throats) ${ }^{4}$

\section{Prevention}

Prevention is currently poorly pursued and would be based on avoiding contact with obvious carriers of cold pathology, frequent hand washing and, when possible, using protective masks by the infected, which, however, until recently was not widespread in western society.

Vaccination is still not easily practicable, due to the fact, that these diseases are attributable to many viruses, which are moreover rapidly changing.

What is almost always implemented, is the use of symptomatic anti-inflammatory and analgesic treatments, to alleviate the symptoms and reduce the duration of the course of the diseases in question. ${ }^{3}$

\section{Remarks}

Analyzing the epidemiology and the clinic of colds, the first question is, why they appear above all in the cold seasons, or in correspondence of periods of sudden thermal decrease and why they affect the upper respiratory tract, and initially, the mucosa of the nasal and paranasal cavities in particular and/or of the oral cavity and the larynx.

That is, because for the most part they replicate better at temperatures of around $33-35^{\circ} \mathrm{C}$ or lower, so they could be defined as "cryophilic" (cold loving).

Therefore they prefer the mucous membranes of the "colder" or "more easily cooled" territories, whose average temperature is lower than the average basal endocorporeal human standard $\left(37^{\circ} \mathrm{C}\right) . .^{3,5,7}$

Rhinoviruses replicate at a temperature of $33^{\circ} \mathrm{C}$, which corresponds to the temperature of the human nasal mucosa. ${ }^{6}$

Rhinoviruses replicate more easily at slightly lower temperatures than the body temperature (around $32 / 33^{\circ} \mathrm{C}$, compared to $37^{\circ} \mathrm{C}$ in our body), which is why they attack the upper airways, and in particular the mucosa of the nose, where they find the ideal climate to multiply. ${ }^{6,8}$

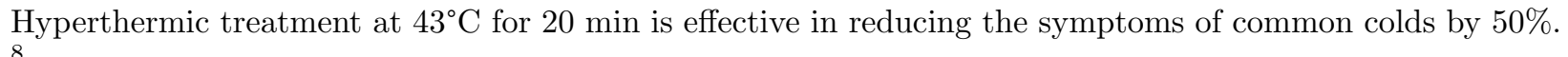

A 20-min hypertermic treatment at $45^{\circ} \mathrm{C}$ is effective in suppressing human Rhinoviruses multiplication by more than $90 \%$ when applied at specific stages of the virus replication cycle. ${ }^{9}$

Coronavirus dried on smooth surfaces retains its vitality for over 5 days at temperatures of $22-25^{\circ} \mathrm{C}$ and a relative humidity of $40-50 \%$, which is typical for air-conditioned rooms. ${ }^{10}$

Conversely, the viability of the virus is rapidly lost $(>3 \log 10)$ at higher temperatures and higher relative humidity (for example: $38^{\circ} \mathrm{C}$ and a relative humidity of $>95 \%$ ). ${ }^{11}$

The improved stability of the SARS-CoV at low temperature and low humidity environment can facilitate its transmission in communities in the subtropical area (such as Hong Kong) in air-conditioned environments. 12

In a experimental 2012 study Xiangyug Q. characterized viral (bacteriofage $\lambda$ ) structure changes upon heating. ${ }^{13}$

Moreover, innate immune defenses have a temperature-dependent efficacy in limiting the replication of common cold viruses: it increases with warm temperature ${ }^{6}$ 
In fact, it is documented that hyperthermia induces endogenous production of $\gamma$-interferon (IFN $\gamma$ ), with a consequent antiviral effect. ${ }^{14,15,16}$

It should certainly be mentioned, that a Cochrane review of 2017 does not detect any difference in results in subjects with acute rhinitis in progress, therapeutically using the treatment with humidified and heated air at $40-47^{\circ} \mathrm{C}$ administered with the RhinoTerm device, compared to the group of control. ${ }^{17}$

These conclusions are also reflected in a subsequent 2018 BMJ paper. ${ }^{18}$

However, these assessments consider the effect of humidified and heated air as a therapy of a disease in progress and not as a preventative treatment.

Vice versa, the Yamaya's 2019 study on the effect of high temperature on pandemic and seasonal human influenza viral replication ${ }^{19}$ and the very recent work (February 2020) published by Wang M. are particularly interesting. In this last paper the difference in the diffusion of the Coronavirus SARS-CoV-2 in different chinese cities is detected, correlating with different relative temperatures. As a result, a minimum temperature increase of $1^{\circ} \mathrm{C}$ correlates with the reduction in the cumulative number of cases by a factor of 0.86 and at a temperature of $38^{\circ} \mathrm{C}$ the virus quickly loses its activity. ${ }^{20}$

Although the spread of the viral airway diseases, in particular of the more serious, such as the recent pandemic from Coronavirus SARS-CoV-2, cannot be influenced only by ambient temperature, the correlations between the spread of virosis and the environmental temperatures are documented. ${ }^{21}$

These epidemiological evaluations are consistent with the potential use of hyperthermic treatment not so much as therapy for acute pathologies, but for prophylactic/preventive purposes.

\section{Prevention proposal}

The viruses in question are ubiquitous and with normal life and relationship, even if only by speaking (direct exchange of contaminated Függe droplets) or by saying goodbye with a handshake, various viral types are acquired by contamination in the upper respiratory tract.

The nose, mouth and face are repeatedly touched with the hands (it is documented, that on average everyone brings the hands to mouth and nose more than 23 times a day), thus favouring the passage of any microbial contaminants from the hands to the upper respiratory tract.

If the environmental conditions of the upper respiratory tract are favourable, due to the relatively low temperature, compared to other body areas, these viruses take root and multiply, up to quantities such as to induce an acute phlogistic response.

Avoiding contamination would be effective as primary prevention, but it is not substantially applied as a daily practice or simply impossible.

However, one could intervene on the contaminating viruses, to reduce their multiplication and engraftment to the nasal, paranasal and upper respiratory tract mucous membrane and thus avoiding the evolution from contamination to infection.

Considering that, as indicated, the main viruses implicated in colds, prefer low temperatures and lose virulence at higher temperatures and considering that, the different parts of the upper respiratory tract (nose, paranasal sinuses, oral cavity) have average temperatures lower than two to three degrees, compared to the core body temperature, the operative proposal therefore consists in warming the upper respiratory tract several times a day, to reduce the viral contaminating share of "cryophilic" viruses, thus reducing or preventing their multiplication. Therefore the evolution from contamination to infection would be interrupted.

This can be simply achieved by heating the nose, jaws, ear cavities and throat by means of a flow of hot air, produced, for example, by a simple hairdryer with at least 600 watts of power.

To generate a flow of hot air with the indicated characteristics, a normal hairdryer can be used. 
The hot air jet is produced by an electric motor that rotates a fan. This fan absorbs and moves air, directing it to an electric heating resistor. The resistor is made up of a twisted nickel-chromium wire wound on a heatresistant support, in which the jet of air is heated and then blown out. A heating resistence with a power consumption of $600 \mathrm{~W}$ is sufficient. The motor can be a permanent magnet DC motor or a single-phase $\mathrm{AC}$ motor. The air flow rate generated by the fan is normally around $40-50 \mathrm{~m}^{3} / \mathrm{h}$. The heating element is protected by a thermostat and the outlet air temperature is around $70-80^{\circ} \mathrm{C}$.

The $70-80^{\circ} \mathrm{C}$ hot air-flow produced by hair dryers easily allows increasing the temperature of the affected areas to over $40^{\circ} \mathrm{C}$.

Such applications, if repeated daily for two minutes, two to four times a day, in the risk seasons (autumnwinter, or in case of sudden decreases of temperature), before the onset of the pathological manifestations of common cold related diseases, allow effective prophylaxis of such affections.

The Messini method suggests the heating of the territories of the nasal cavities, of the areas of the frontal and maxillary sinuses, followed by the jaws with open mouth, subsequently of right and left ear, finally the right and left lateral cervical region.

The procedure should be performed at least two to four times daily for at least two minutes, over the whole cold season. In epidemic periods it should be repeated several times a day.

Such treatment can contrast the "vital" and replicative biological capacities of the "cryophilic" viruses.

Limits of the proposed method of thermal prevention according to Messini of common cold diseases

Obviously the result can only be obtained with a regular application of the method and, therefore, it is intrinsically subjective and dependent on individual diligence.

The availability of a small pocket device (e.g. 110x40 mm), capable of producing such a flow of hot air, could make the repetitiveness of the procedure more easily practicable.

At the moment there are no randomized prospective studies that certify the effectiveness of the proposed preventive method, but only personal observational case studies, as a pilot evaluation.

Two studies would be scheduled in this regard:

a) A laboratory study with exposure to different thermal gradients of cultures of viral material taken from the airways of affected subjects, to verify if and at what levels the elevation of the temperature actually has a negative impact on the viral "vitality"

b) A longitudinal single blind clinical-observational study over several months between two homogeneous groups of selected volunteers: a proband group (which regularly applies the method) and a control group (which does not apply it); the evaluation at the end of the observational period would allow, to confirm a possible statistically significant difference in the frequency of occurrence of the symptoms of common cold in the two groups.

\section{Conclusion}

Considering the biology of the spread of the viruses causing common cold and/or upper respiratory tract diseases, Rhinovirus, Coronavirus, Orthomyxovirus in primis, the Thermo-Prevention Method proposed in this paper, avoiding the evolution from contamination to infection, appears to be conceivable, as an effective means of additional self-prevention.

The aim of this presentation, is to stimulate interest in this simple method of prevention and to promote the collaboration for the design of larger studies.

This could be even more interesting in relation to the COVID-19 pandemic of the year 2020, pending the development of a specific vaccination coverage. 


\section{Conflict of interest}

The authors declare that there is no conflict of interest.

\section{REFERENCES}

1) Hagen C.

Economic impact of cold virus to be more expensive than asthma, heart failure

University of Michigan

The university record online - 10.03.2003

2) Fendrick A.M. et alii

The economic burden of non-influenza-related viral respiratory tract infection in the United States

Arch. Intern. Med. 2003; 164(4): 487-94

3) Nuove evidenze sulla prevenzione del raffreddore comune

Obiettivo ECM - September 2009

4) Jacobs Samantha E. et alii

Human Rhinoviruses

2013; 26(1): 135-161

5) Redazione MyPersonalTrainer

Virus del raffreddore

Mondadori Media SpA - 27.01.2020

6) Papadopulos N. G. Et alii

Rhinoviruses replicate effectively at lower airway temperatures

Wiley Online Library Journal of Medical Virology 1999; 58(1)

7) Foxman EF et alii

Temperature-dependent innate defense against the common coldvirus limits viral replication at warm temperature in mouse airway cells

Proc Natl Sci USA 2015 Jan 20; 112(3): 827-32

8) Tyrrel David et alii

Local Hyperthermia benefits natura ad experimenatle common colds

British Medical Journal, 1989; 298: 1280-3

9) Conti C. et alii

Antiviral Effect of Hyperthermic Treatment in Rhinovirus Infection

Antimicrobial Agents and Chemotherapy 1999; 43 (4):822-29

10) Ikäheimo T.M., et alii

A decrease in temperature and humidity precedes human rhinovirus infection in a cold climate

Viruses 02 Sept 2016

11) Casanova L.M. et alii 
Effects of air temperature and releative umidity on coronavirus survival on surfaces

Am. Soc Microbiol. Applied and environmental microbiology 2010; 76(9): 2712-17

12) Chan K.H. et alii

The effects of temperature and relative humidity in the viability of SARS Coronavirus

Hindawi Publishing Corporation Advances in Virology 2011, Article ID 734690

13) Xiangyun Qiu

Heat Induced Capsid Disassembly and DNA Release of Bacteriophage $\lambda$

PLoS ONE (www.plosone.org) July 2012; Volume 7 Issue 7: e39793

14) Downing JF at alii

In vivo hyperthermia enhances plasma antiviral activity and stimulates peripheral lymphocites for increased synthesis of interferon- $\gamma$

Journal of Interferon research 2009; 7 (2)

15) Zhu L et alii

Local Hyperthermia could induce antiviral activity by endogenous interferon-dependet pathway in condylomata acuminata

Antiviral research 2010; 88:187-92

16) Mace Thomas A. et alii

Effector CD8 T cell IFN production and cytotoxicity are enhanced by mild hyperthermia

International Journal of Hyperthermia 2012; 28:1, 9-18

17) Singh M. et alii

Heated, humidified air for the common cold

Cochrane database and systematic review 2017 (8); Art.No.:CD001728

18) van Driel N.L. et alii

What treatments are effective for common cold in adults and children?

BMJ 2018; 363: k3786

19) Yamaya M. et alii

Effect of high temperature on pandemic and seasonal human influenza viral replication and infection-induced damage in primary human tracheal epithelial cell coltures

Heliyon 2019 Feb; 5(2): e01149

20) Wang Mao et alii

Temperature significantly changes COVID-19 transmission in 429 cities

medR $\chi$ iv The reprint server for healt sciences - 22.02.2020 doi.org/10.1101/2020.02.22.20025791

21) Bukhari Qasim and Jameel Yusuf

Will Coronavirus Pandemic Diminish by Summer? (March 17, 2020). Available at SSRN:https://ssrn.com/abstract=3556998 orhttp://dx.doi.org/10.2139/ssrn.3556998 